\title{
Survey on Android Based Live Monitoring System of Green House Parameter
}

\author{
Jayashri G. Hagaone ${ }^{1}$, M. D. Jakhete ${ }^{2}$ \\ ${ }^{1}$ PG Student, Electronics, M.E. Digital Electronics, GHRIEM, Jalgaon, India \\ ${ }^{2}$ Assistant Professor, Department of E \& Tc Engineering, GHRIEM. Jalgaon, India
}

\begin{abstract}
Agriculture is the worldwide prime occupation of human being.There is a strong correlation between agricultural growth and economic growth. We need a new and effective technology which can improve continuously the productivity, profitability of our major farming systems. In earlier method of farming farmer were required to visit their farm repeatedly. This is very time saving $g$, and it required lots of work and effort. This system focus on automatic monitoring of greenhouse parameters using wireless sensor network using embedded system. In this project Raspberry Pi is used as an embedded Linux board whose designed is based on the arm 9 microcontroller architecture. Embedded Linux board makes the communication between all distributed sensor nodes placed in the farm through ZigBee protocol and itself act as a coordinated node in the wireless sensor network. The main work of coordinator node is to collect the parameters like soil moisture and soil temperature wirelessly. Each sensor node consists of soil moisture and soil temperature sensor and ZigBee RF antenna device for communication with the coordinator node. Raspberry Pi stores collected data in the database and analyzes the stored data. The system will work according to the algorithm developed for watering the crop. In the proposed system project monitoring is done with the help of android mobile phone. In software design embedded $C$ is used for coding. With the help of this system Irrigation monitoring is done remotely with the help of Android mobile phone.
\end{abstract}

Keyword:ARM Processor, raspberry Pi, Arduino Device

\section{Introduction}

Green house monitoring system is technical intransitive in which the farmer in the rural area is benefited by automatic monitoring and control of greenhouse environment greenhouse is the building where the plant can grows in proper manner. Agriculture is the worldwide prime occupation of human being. For proper growth proper supply of water must be required. Means water given to the plant must be in sufficient proportion. Irrigation is the process of artificial supply of water to the land or soil. Good irrigation water management will increases yield, improves crop quality, conserve water, saves energy, conserve fertilizer requirement and reduce non-point source pollution. The most important factors for the quality and productivity of plant growth are temperature, humidity, light level, level of carbon-dioxide. Continuous monitoring and recording of these environmental variables gives information to the grower to better understand. Wireless system can form a useful part of automation system architecture in modern greenhouses. With the use of greenhouse concept, the farmer can produce different crops in different climates and various seasons. The agro-environmental remote monitoring method can be implemented in various situations such as monitoring qualities of soil and water. However this research focuses independently in remotely monitoring levels of temperature in greenhouse. By using proper technology, the natural environment and resource, in this case is temperature, which is very important for the plants and can be monitored in good manner. Previously, human labour plays a very important role in monitoring farm and plants in this industry. For some plants such as vegetables and flowers, they need 24 hours attention from human so that the qualities of the plant can be controlled. With proper management of collected data and information, this project will provide them with strong foundation for future development and future growth of the plants. However, because of increasing size in farm areas, this type of manual practice is apparently time consuming and labour-demanding. However, with the improvement of management in agriculture techniques, modern telecommunication technologies can be implemented which provide great subordinate for the industry. Due to the fast improvement in telecommunication technologies, it is believed that wireless communication is a good practice for remote judgment in the agriculture industries. This research has fully utilized wireless sensor network, Global System for Mobile Communication (GSM) and short message service (SMS) to carry out data from the sensors to computers or directly alert the workers through their mobile phone. The proposed system is aimed to be a reliable and cost effective. There are a many technologies that have been created to perform the operation; however, many of the existing technologies would still require a great deal of human interfacing. A part from that, existing technology for detecting level of temperature this works is based on reactive measurement whereby it only alert workers when the levels reach at certain values. In proposed system of design of the green house, the farmer can easily keep the desired crop's Environment conditions. To obtain this requirement we need the environmental parameter sensors, such as Temperature sensor, humidity sensor, $\mathrm{CO} 2$ sensor etc. The use of wireless sensor network for the large area is now becoming popular in greenhouse technology. All these sensors are connected to the server with the help of link without use of wire, hence such network is called as wireless sensor network (WSN). Wireless communication can be used to collect the measurement and to communicate between the centralized control systems which ARM processor. Wireless Sensors Network maintenance is relatively cheap and easy. A ZigBee sensor network can use to maintain network performance at a high level. The only additional costs occur when the sensor nodes run out of batteries these batteries need to be charged or replaced, but the lifespan of battery

\section{Volume 4 Issue 12, December 2015}




\section{International Journal of Science and Research (IJSR) \\ ISSN (Online): 2319-7064}

Index Copernicus Value (2013): 6.14 | Impact Factor (2014): 5.611

can be many years if an efficient power saving algorithm is applied. By using these sensors, we are able to measure four Parameters, which are essential in greenhouse climate adjustment: temperature, relative humidity, light irradiance and air carbon dioxide content.

\section{Related Work}

Jia Song[1] He designed a system on Greenhouse Monitoring and Control System Based on Zigbee Wireless Senor Network using 8051 controller. In this paper they converse about Greenhouse Monitoring and Control System Based on Zigbee Wireless Senor Network using ARM controller and it has ability of being used through the Internet. Ai,Wei,Chen, Cifa [2] They have discussed about Green House environment monitor technology implementation based on android platform, which uses android cell phone as the recording terminal. In this paper parameters in the greenhouse are monitored on the computer as well as the android cell phone from anyplace on the world as it is connected to the internet through the team viewer software. Guomi Wang, Xiao Chan; Sun, Guoxiang [3].They had discussed about humidity and moisture monitoring in green house with the help of Zigbee monitoring system. In this paper we have proposed a low cost method using ARM microcontroller and Zigbee technology to correctly monitor and control the various parameters like humidity, soil moisture, light intensity and temperature. Aryo $\mathrm{H}$. Primicanta, Mohd Yunus Nayan, and Mohammad wan[4] they proposed a method on ZigBee-GSM which is based on Automatic Meter Reading system. In this paper we are using GSM modem to transmit fault messages to user via Zigbee.

The green house monitoring system is based on Wireless Sensor Network is designed to monitor environmental factors for urban green house. By using a Wireless Sensor Network, the real-time distribution of temperature and humidity gathered from the real environment in clear manner and reflects the dynamics of temperature and humidity both inside and outside the green house. Meanwhile, the data of the heat occupies and the humidity released by urban greenhouse can also be obtained. Finally, it is discovered that temperature and humidity were different inside and outside the urban green house, the green house has a good effect on cooling and humidity, and the trend of the changes in the temperature and humidity is gradually penetrating from east to west. The WSN based automatic control system to prevent dew condensation in the greenhouse environments has come up with the solution for different problem. This system is composed with the help of sensor nodes for collecting data, base nodes for processing collected data, relay nodes for settling the environment inside a greenhouse and an environment server for storage and processing of collected data. With the help of the Barenbrug formula for calculating the dew point on the leaves, this system is realized to prevent a moisture condensation phenomenon on the crop's surface acting as an important element in diseases generation. They also constructed a setup for the usual greenhouse in order to verify the performance of their system with regard to moisture condensation control. An Embedded systems approach to Monitor Green House is an approach to measure parameters in the Green house.
They have designed a system which consists of sensor circuits, PIC microcontroller, RS 232 serial communication, LCD module to display the parameters. The GSM modem is used to update user, mobile receiver, and required power supply unit. The output of the sensors is given as input to the micro controller to control, display the parameters and update the user. Any parameter is change with set parameter for Green House systems, the micro controller will read and stores periodically, and it updates the user by sending SMS by service provider. The combination of WSN and fuzzy control is aiming at the features of environmental monitoring, a multi-parameter monitoring system is designed by Xinrong and Chang based on ZigBee wireless communication technology to improve overall the level of system automation and monitoring. The monitoring results of temperature and humidity have shown that this system is stable, highly suitable in data transmission and is easy to use, and can be widely used in various areas of automatic monitoring of environmental parameters. Another practical energy saving method was constructed in different environment to verify the efficiency of the system. This research is apply in new technology to build up active \& intelligent energy-saving system focus on system operation as substitute of materials or components, with those autodetect mechanism and auto-judged back-end agent software, then gives feedback control signals through bi-direction wireless communication interface, this system can effectively obtain the goal of energy-saving. Green house environment monitor technology implementation based on android mobile platform is an advanced technology introduced by different kind of monitoring system. In this paper they use mobile cell phone as monitoring terminal, monitoring green-house environment. Recently, intelligent systems of agricultural production are being implemented for safe and low cost food production. Plant factory provide high crop by growing multiple crops and making efficient use of land and resources. Plant growth is easily obtained by maintaining humidity, temperature, $\mathrm{CO} 2$ proportion and light intensity and these factors need to be monitored an automated system. In this paper, they have proposed a control system for a LED based plant factory consist of ZigBee wireless mesh network, and remote monitoring via Internet. A major novelty of the system is the use of LED lighting is used instead of fluorescent lighting due to its low power consumption, long life and useful narrow band. LED lighting system provides a proper and economical lighting system that facilitates plant growth by varying light intensity and frequency corresponding to light conditions and growing requirements and also helps in reducing production costs and speeding growth.

\section{Proposed System Architecture}




\section{International Journal of Science and Research (IJSR) \\ ISSN (Online): 2319-7064}

Index Copernicus Value (2013): 6.14 | Impact Factor (2014): 5.611

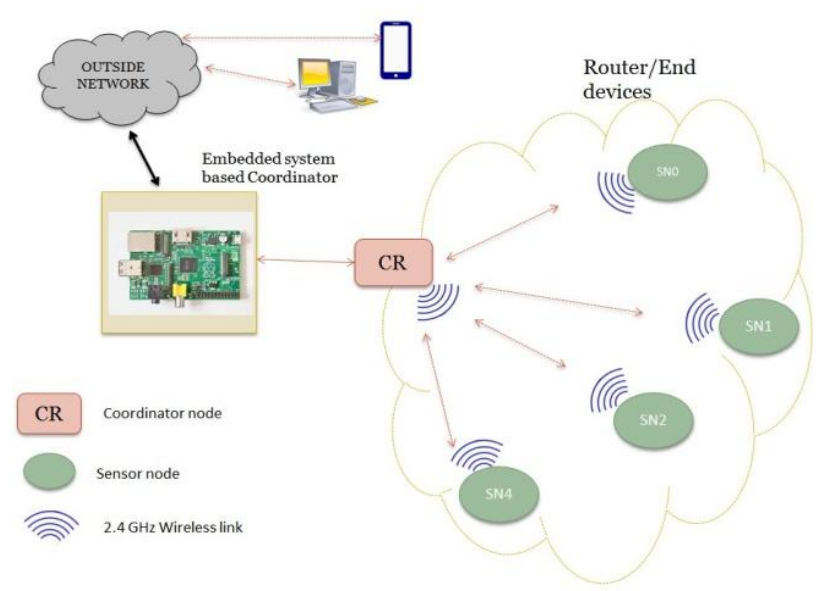

Figure 1: Architecture of the system

a) Raspberry Pi-

In the proposed system we used the raspberry pi as controller. Which is based on ARM 9 architecture. It is the core of the system.

b)Zigbee -

A ZigBee protocol for wireless communication which is based on the underlying protocol IEEE 802.15.4. ZigBee protocol defines the network layer, application layer and specifications of the network security services.

c) Sensors -

Soil moisture sensors - The module consists of, detection probe, and sensor board. It is having triple output mode, digital, analog, and serial with exact readings. The sensor will detect the moisture of the soil surrounding it, i.e. shortage of water content of the soil.

Soil temperature sensors - This sensor consist of digital sensor, to measure soil temperature.

d) Android mobile -

Android mobile phone is used to operate or monitoring the greenhouse parameter. Such as temperature, moisture, humidity of the soil.

\section{Conclusion}

This paper designs the automated wireless irrigation system using WSN and embedded Linux board. In this we have used raspberry $\mathrm{Pi}$ as an embedded Linux board which allows collecting the sensor information from sensor node continuously, store it in a database and providing the web interface to the user. The system is watering to the crop uniform by analysing the soil parameters, it will help to reduce the fresh water consumption. By providing the web interface and automation user can easily monitor the system and it will minimize the human intervention. The ZigBee protocol is used here for wireless communication it will create network easily and combination of Arduino, Xbee and sensor create a low power inexpensive sensor node. The Apache web server crated on Raspberry Pi easily displaying the contents of sensor data. In this project automated greenhouse control is discussed with advantages of low cost and accuracy.

This system is provided with less manpower and many other several benefits .This system only give water to crop only when moisture level of soil is goes below the certain point. With the help of this system water supply can be given directly to the root of the plant this saves water conservation. This system is very much compactable for changing and saving environmental conditions.

\section{References}

[1] Jian Song, "Greenhouse Monitoring and Control System Based on Zigbee Wireless Senor Network" ICECE '10 Proceedings of the 2010 International Conference on Electrical and Control Engineering IEEE Computer Society Washington, DC, pp.2785-2788 USA (C2010.

[2] Ai, Wei,Chen,Cifa," Greenhouse environment monitor technology implementation based on android mobile platform" Artificial Intelligence, Management Science and Electronic Commerce (AIMSEC),2011 2nd International Conference.

[3] He,Guomi Wang, Xiaochan; Sun, Guoxiang, " Design of a Greenhouse Humiture Monitoring System Based on ZigBee Wireless Sensor Networks "Fifth International Conference on Frontier of ComputerScience and Technology (FCST), 2010

[4] Aryo H. Primicanta, Mohd Yunus Nayan, and MohammedAwan, "ZigBee-GSM based Automatic Meter Reading System", IEEE International conference on Digital Object Identifier, pp. 1-5, 2010

[5] Shuo Liu, Guomo Zhou, Lufeng Mo , The green space monitoring system based on Wireless Sensor Network Education Technology and Computer (ICETC), 2010 2nd International Conference, volume 5, 2010, Pages: V5- 180 - V5-183.

[6] DaeHeon Park, ChulYoung Park, SungEon Cho, JangWoo Park, Greenhouse Environment Monitoring and Automatic Control System Based on Dew Condensation Prevention, Embedded and Multimedia Computing (EMC) 2010, 5th International Conference. 